\title{
Robust feedback stability of negative imaginary systems: An integral quadratic constraint approach
}

\author{
Sei Zhen Khong, Ian R. Petersen, and Anders Rantzer
}

\begin{abstract}
Sufficient conditions for stability of feedback interconnections of negative imaginary systems are derived via an integral quadratic constraint (IQC) approach. These extend existing results in the literature by exploiting the flexibility present at the static and infinite frequencies to reduce conservatism. Negative imaginary transfer functions with poles on the imaginary axis are accommodated using a recently generalised IQC-based robustness result. In particular, the negative imaginary property of systems is shown to give rise to IQCs on positive frequencies that are bounded away from zero and infinity. Additional quadratic conditions are introduced to take care of the IQCs near the DC and instantaneous gains of the systems.
\end{abstract}

Index Terms-Negative imaginary systems, feedback stability, integral quadratic constraints

\section{INTRODUCTION}

The notion of negative imaginary systems was introduced in [1], [2]. The negative imaginary property commonly arises from the dynamics of a lightly damped structure with colocated force actuators and position sensors (such as piezoelectric sensors) [3], [2]. Such a system exhibits positive real dynamics [4] from the force input to the velocity output, but negative imaginary dynamics from the force input to the position output, whose transfer function may be of relative degree 2, rendering the standard positive real results inapplicable. Another area where negative imaginary dynamics are found is that of nano-positioning systems [5].

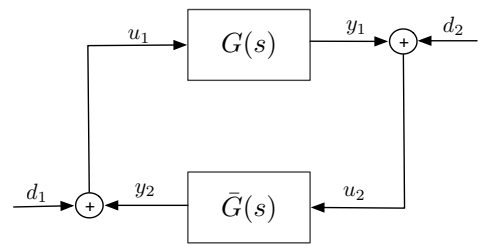

Fig. 1. Positive feedback of negative imaginary systems.

Due to the prevalence of negative imaginary properties in real world applications, the robustness of feedback interconnections of open-loop stable negative imaginary systems is investigated in [1] as a parallel to the positive real stability results [4]; see Figure 1. It is shown that if the instantaneous

This work was supported by the Swedish Research Council through the LCCC Linnaeus centre and the Australian Research Council.

S. Z. Khong and A. Rantzer are with the Department of Automatic Control, Lund University, SE-221 00 Lund, Sweden. \{seizhen; anders.rantzer\}econtrol.lth.se

I. R. Petersen is with the School of Engineering and Information Technology, University of New South Wales Canberra at the Australian Defence Force Academy, Canberra ACT 2600, Australia. i.r.petersen@gmail.com gain of $\bar{G}$ is positive semidefinite, i.e. $\bar{G}(\infty) \geq 0$, and the product of the instantaneous gains of $\bar{G}$ and $G$ is 0 , then the closed-loop system $[\bar{G}, G]$ is internally stable if, and only if, the DC gain condition $\bar{\lambda}(G(0) \bar{G}(0))<1$, where $\bar{\lambda}$ denotes the maximum eigenvalue. This result is further generalised in [6] to the case where $G$ may have imaginary-axis poles that are not located at the origin. These stability conditions are robust in the sense that they are invariant to negativeimaginary perturbations on the systems, provided that the aforementioned gain conditions are not destroyed. Stability conditions for negative imaginary systems with poles at the origin are studied in [7].

When the presuppositions of the stability theorems in [1], [6] do not hold, such as $\bar{G}(\infty)$ being sign-indefinite, then the DC gain condition may not be necessary. This paper derives generic sufficient conditions for feedback stability of stable negative imaginary systems using the theory of integral quadratic constraints (IQC) [8], [9]. In particular, it is established that the negative imaginary properties of the systems give rise to complementary IQCs on a compact set of frequencies which do not include 0 and $\infty$ but can be arbitrarily large. This interpretation clarifies clearly the role of negative imaginariness in IQC analysis. Furthermore, it leads to the observation that feedback stability follows if there exist constant multipliers such that the corresponding complementary IQCs hold at 0 and $\infty$. The robust stability result is shown to extend to negative imaginary systems that are only marginally stable, i.e. have poles on the imaginary axis. To this end, a recently developed notion of IQCs for marginally stable systems from [10] is employed to conclude closed-loop stability. It is noteworthy that a relevant work on mixed passivity, negative imaginary, and small gain properties, all of which are characterisable by IQCs, can be found in [11].

The paper evolves along the following lines. Robust stability of feedback interconnections of stable negative imaginary systems is considered in Section II. Section III reviews a recent result on IQC conditions for concluding a generalised notion of feedback stability. This is then utilised to derive robust stability conditions for negative imaginary systems with imaginary-axis poles in Section IV. A couple of numerical examples are given in Section V to illustrate the theory. Finally, concluding remarks are provided in Section VI.

\section{StABlE NEGATIVE IMAGINARY TRANSFER FUNCTIONS}

In this section, sufficient conditions which guarantee closed-loop stability of stable negative imaginary systems are 
derived. The proof methods in this section will be reused in the subsequent section where systems with imaginaryaxis poles are accommodated. It begins by introducing the notation used in this paper.

$\mathbb{R}$ and $\mathbb{C}$ denote, respectively, the real and complex numbers. $\mathbb{C}_{+}$denotes the open right half plane and $\overline{\mathbb{C}}_{+}$its closure. Let $\mathcal{R}^{n \times m}$ denote the set of real-rational proper transfer function matrices of dimensions $n \times m$ and $\mathcal{R} \mathbf{H}_{\infty}{ }^{n \times m}$ denote the subset of stable transfer functions in $\mathcal{R}^{n \times m}$. Define

$$
\begin{aligned}
\hat{\mathbf{N}}:=\{ & R \in \mathcal{R} \mathbf{H}_{\infty}{ }^{n \times n}: \\
& \left.j\left[R(j \omega)-R(j \omega)^{*}\right] \geq 0 \forall \omega \in(0, \infty)\right\} \\
\mathbf{N}_{s}:=\left\{R \in \mathcal{R} \mathbf{H}_{\infty}{ }^{n \times n}:\right. & \\
& \left.j\left[R(j \omega)-R(j \omega)^{*}\right]>0 \forall \omega \in(0, \infty)\right\} \subset \hat{\mathbf{N}} .
\end{aligned}
$$

$\hat{\mathbf{N}}$ denotes the set of stable negative imaginary transfer functions; a more general definition accommodating marginally stable poles will be given in the next section. $\mathbf{N}_{s}$ denotes the set of strictly negative imaginary transfer functions. An $R \in \hat{\mathbf{N}}$ satisfies $R(0)=R(0)^{T} \in \mathbb{R}^{n \times n}$ and $R(\infty)=$ $R(\infty)^{T} \in \mathbb{R}^{n \times n}$ [1, Lem. 2]. As such, it follows that $j\left[R(j \omega)-R(j \omega)^{*}\right]=0$ when $\omega=0$ or $\omega=\infty$.

Denote by $\bar{\sigma}(A)$, the largest singular value of matrix $A$ and by $\bar{\lambda}(B)$, the largest eigenvalue of a Hermitian matrix $B=B^{*}$.

Theorem 2.1: Given $G \in \hat{\mathbf{N}}$ and $\bar{G} \in \mathbf{N}_{s}$, suppose there exist $\Pi_{0}=\Pi_{0}^{*} \in \mathbb{C}^{2 n \times 2 n}, \Pi_{\infty}=\Pi_{\infty}^{*} \in \mathbb{C}^{2 n \times 2 n}$ such that for some $\epsilon>0$ and all $\tau \in[0,1]$,

$$
\begin{gathered}
{\left[\begin{array}{c}
\bar{G}(0) \\
I
\end{array}\right]^{*} \Pi_{0}\left[\begin{array}{c}
\bar{G}(0) \\
I
\end{array}\right]} \\
{\left[\begin{array}{c}
I \\
\tau G(0)
\end{array}\right]^{*} \Pi_{0}\left[\begin{array}{c}
I \\
\tau G(0)
\end{array}\right]}
\end{gathered}
$$

and

$$
\begin{gathered}
{\left[\begin{array}{c}
\bar{G}(\infty) \\
I
\end{array}\right]^{*} \Pi_{\infty}\left[\begin{array}{c}
\bar{G}(\infty) \\
I
\end{array}\right]} \\
{\left[\begin{array}{c}
I \\
\tau G(\infty)
\end{array}\right]^{*} \Pi_{\infty}\left[\begin{array}{c}
I \\
\tau G(\infty)
\end{array}\right] \geq 0}
\end{gathered}
$$

Then the feedback interconnection $[\bar{G}, G]$ is internally stable.

Proof: $\quad$ Let $\hat{\Pi}_{0}:=2 \Pi_{0}+\epsilon I$ and $\hat{\Pi}_{\infty}:=2 \Pi_{\infty}+\epsilon I$. Then the inequalities above are equivalent to

$$
\left[\begin{array}{c}
\bar{G}(0) \\
I
\end{array}\right]^{*} \hat{\Pi}_{0}\left[\begin{array}{c}
\bar{G}(0) \\
I
\end{array}\right] \leq-\epsilon I ; \quad\left[\begin{array}{c}
I \\
\tau G(0)
\end{array}\right]^{*} \hat{\Pi}_{0}\left[\begin{array}{c}
I \\
\tau G(0)
\end{array}\right] \geq \epsilon I
$$

and

$$
\begin{gathered}
{\left[\begin{array}{c}
\bar{G}(\infty) \\
I
\end{array}\right]^{*} \hat{\Pi}_{\infty}\left[\begin{array}{c}
\bar{G}(\infty) \\
I
\end{array}\right]} \\
{\left[\begin{array}{c}
I \\
\tau G(\infty)
\end{array}\right]^{*} \hat{\Pi}_{\infty}\left[\begin{array}{c}
I \\
\tau G(\infty)
\end{array}\right] \geq \epsilon I}
\end{gathered}
$$

for all $\tau \in[0,1]$. By continuity of rational transfer functions, the hypothesis implies there exist sufficiently small $\underline{\Omega}>0$ and sufficiently large $\bar{\Omega}>0$ such that

$$
\begin{aligned}
{\left[\begin{array}{c}
\bar{G}(j \omega) \\
I
\end{array}\right]^{*} \hat{\Pi}_{0}\left[\begin{array}{c}
\bar{G}(j \omega) \\
I
\end{array}\right] } & \leq-\frac{\epsilon}{2} I ; \\
{\left[\begin{array}{c}
I \\
\tau G(j \omega)
\end{array}\right]^{*} \hat{\Pi}_{0}\left[\begin{array}{c}
I \\
\tau G(j \omega)
\end{array}\right] } & \geq \frac{\epsilon}{2} I
\end{aligned}
$$

for all $\omega \in[0, \underline{\Omega}], \tau \in[0,1]$ and

$$
\begin{gathered}
{\left[\begin{array}{c}
\bar{G}(j \omega) \\
I
\end{array}\right]^{*} \hat{\Pi}_{\infty}\left[\begin{array}{c}
\bar{G}(j \omega) \\
I
\end{array}\right] \leq-\frac{\epsilon}{2} I} \\
{\left[\begin{array}{c}
I \\
\tau G(j \omega)
\end{array}\right]^{*} \hat{\Pi}_{\infty}\left[\begin{array}{c}
I \\
\tau G(j \omega)
\end{array}\right] \geq \frac{\epsilon}{2} I}
\end{gathered}
$$

for all $\omega \in[\bar{\Omega}, \infty], \tau \in[0,1]$. Now note from the definitions of $\mathbf{N}$ and $\mathbf{N}_{s}$ that $G \in \hat{\mathbf{N}}$ and $\bar{G} \in \mathbf{N}_{s}$ implies that there exists $\eta>0$ such that

$$
\begin{gathered}
{\left[\begin{array}{c}
\bar{G}(j \omega) \\
I
\end{array}\right]^{*} \Pi_{m}\left[\begin{array}{c}
\bar{G}(j \omega) \\
I
\end{array}\right]} \\
{\left[\begin{array}{c}
I \\
\tau G(j \omega)
\end{array}\right]^{*} \Pi_{m}\left[\begin{array}{c}
I \\
\tau G(j \omega)
\end{array}\right]}
\end{gathered}
$$

for all $\tau \in[0,1]$ and $\omega \in[\underline{\Omega}, \bar{\Omega}]$, where

$$
\Pi_{m}:=\left[\begin{array}{cc}
0 & j I \\
-j I & 0
\end{array}\right] .
$$

Let $\hat{\Pi}_{m}:=2 \Pi_{m}+\eta I$, then

$$
\begin{gathered}
{\left[\begin{array}{c}
\bar{G}(j \omega) \\
I
\end{array}\right]^{*} \hat{\Pi}_{m}\left[\begin{array}{c}
\bar{G}(j \omega) \\
I
\end{array}\right]} \\
{\left[\begin{array}{c}
I \\
\tau G(j \omega)
\end{array}\right]^{*} \hat{\Pi}_{m}\left[\begin{array}{c}
I \\
\tau G(j \omega)
\end{array}\right] \geq \eta I}
\end{gathered}
$$

for all $\tau \in[0,1]$ and $\omega \in[\underline{\Omega}, \bar{\Omega}]$. Define

$$
\begin{gathered}
\gamma_{0}(j \omega):= \begin{cases}1 & \omega \in[0, \underline{\Omega}] \\
0 & \text { otherwise },\end{cases} \\
\gamma_{\infty}(j \omega):= \begin{cases}1 & \omega \in[\bar{\Omega}, \infty] \\
0 & \text { otherwise }\end{cases}
\end{gathered}
$$

and

$$
\Pi(j \omega):=\gamma_{0}(j \omega) \hat{\Pi}_{0}+\hat{\Pi}_{m}+\gamma_{\infty}(j \omega) \hat{\Pi}_{\infty}, \omega \in[0, \infty] .
$$

Combining (3), (4), and (5) yields that

$$
\begin{gathered}
{\left[\begin{array}{c}
\bar{G}(j \omega) \\
I
\end{array}\right]^{*} \Pi(j \omega)\left[\begin{array}{c}
\bar{G}(j \omega) \\
I
\end{array}\right]} \\
{\left[\begin{array}{c}
I \\
\tau G(j \omega)
\end{array}\right]^{*} \Pi(j \omega)\left[\begin{array}{c}
I \\
\tau G(j \omega)
\end{array}\right]}
\end{gathered}
$$

for all $\omega \in[0, \infty], \tau \in[0,1]$ and some $\zeta>0$. Stability of $[\bar{G}, G]$ then follows from the theory of IQCs [8, Thm. 1] or [12, Thm. 7]; see also Proposition 3.1 with $\epsilon=0$ in the next section.

Remark 2.2: Theorem 2.1 is proven by fabricating a 3part multiplier $\Pi$ in (6) in such a way that the standard IQC result can be applied to conclude closed-loop stability. In particular, the fact that $G \in \hat{\mathbf{N}}$ and $\bar{G} \in \mathbf{N}_{s}$ implies the complementary IQC inequalities hold for positive fre- 
quencies that are bounded from zero and infinity; see (5). The additional matrix inequalities (1) and (2) in the theorem imply the complementary IQC inequalities for sufficiently small and sufficiently large frequencies, i.e. (3) and (4) respectively.

Corollary 2.3: Given $G \in \hat{\mathbf{N}}$ and $\bar{G} \in \mathbf{N}_{s}$, suppose $\bar{\sigma}(G(0) \bar{G}(0))<1$ and $\bar{\sigma}(G(\infty) \bar{G}(\infty))<1$, then the feedback interconnection $[\bar{G}, G]$ is internally stable.

Proof: The hypothesis is equivalent to $\bar{\lambda}\left(\bar{G}(j \omega)^{*} G(j \omega)^{*} G(j \omega) \bar{G}(j \omega)\right)<1$ for $\omega=0$ and $\omega=\infty$. It follows that the matrix inequalities (1) and (2) in Theorem 2.1 hold with respect to

$$
\Pi_{0}:=\left[\begin{array}{cc}
G(0)^{*} G(0) & 0 \\
0 & -I
\end{array}\right]
$$

and

$$
\Pi_{\infty}:=\left[\begin{array}{cc}
G(\infty)^{*} G(\infty) & 0 \\
0 & -I
\end{array}\right],
$$

as required.

\section{GENERALISED IQC CONDITIONS}

In order to accommodate negative imaginary transfer functions with imaginary-axis poles, a generalised version of the IQC result is needed. This section provides an overview of such a generalisation from [10].

Given an $\epsilon>0$ and a point $j q \in j \mathbb{R}$, define the semi-circle of radius $\epsilon$ in the right-half plane as

$$
\mathcal{S}_{\epsilon}(j q):=\{s \in \mathbb{C}:|s-j q|=\epsilon, \Re(s)>0\}
$$

and $\mathcal{S}_{0}(j q):=\{\}$. Given a finite ordered set $j \mathcal{Q}=$ $\left\{j q_{1}, j q_{2}, \ldots, j q_{K}\right\} \subset j \mathbb{R}$ with $q_{1}>q_{2}>\ldots>q_{K}$, define a contour parameterised by $\epsilon \geq 0$ as

$$
\begin{aligned}
\mathcal{C}_{\epsilon}(j \mathcal{Q}):=j\left[q_{1}+\epsilon, \infty\right) & \cup \mathcal{S}_{\epsilon}\left(j q_{1}\right) \cup j\left[q_{2}+\epsilon, q_{1}-\epsilon\right] \\
& \cup \mathcal{S}_{\epsilon}\left(j q_{2}\right) \cup j\left[q_{3}+\epsilon, q_{2}-\epsilon\right] \\
\vdots & \\
& \cup \mathcal{S}_{\epsilon}\left(j q_{K}\right) \cup j\left(-\infty, q_{K}-\epsilon\right] .
\end{aligned}
$$

that is, a straight line on the imaginary axis indented to the right of every point in $j \mathcal{Q}$ by a semi-circle of radius $\epsilon$. In particular, notice that $C_{0}(j \mathcal{Q})=j \mathbb{R}$ for any $j \mathcal{Q} \subset j \mathbb{R}$. Denote by $\mathcal{C}_{\epsilon}^{+}(j \mathcal{Q})$ the open half plane that lies to the right of $\mathcal{C}_{\epsilon}(j \mathcal{Q})$ defined in (8), i.e.

$\mathcal{C}_{\epsilon}^{+}(j \mathcal{Q}):=\left\{s=\sigma+j \omega \in \mathbb{C} \mid \bar{\sigma}+j \omega \in \mathcal{C}_{\epsilon}(j \mathcal{Q}) \Longrightarrow \sigma>\bar{\sigma}\right\}$,

and $\overline{\mathcal{C}}_{\epsilon}^{+}(j \mathcal{Q})$ its closure. The following result can be established as in [10, Thm. 4.4 and 4.5].

Proposition 3.1: Given $\Delta \in \mathcal{R}^{n \times m}$ and $G \in \mathcal{R}^{m \times n}$, where $\Delta$ and $G$ have no poles on $\overline{\mathcal{C}}_{+} \backslash j \mathcal{Q}$, the closed-loop transfer function

$$
\left[\begin{array}{cc}
I & -G \\
-\Delta & I
\end{array}\right]^{-1}
$$

of the feedback interconnection $[\Delta, G]$ has no poles on $\overline{\mathcal{C}}_{+} \backslash j \mathcal{Q}$ if there exists a bounded $\Pi: j \mathbb{R} \cup\{\infty\} \rightarrow$ $\mathbb{C}^{(n+m) \times(n+m)}$ such that and $\Pi(j \omega)=\Pi(j \omega)^{*}$ for all $\omega \in[0, \infty]$ and the following complementary IQC conditions hold:

(i) for all $\omega \in[0, \infty] \backslash \mathcal{Q}$,

$$
\left[\begin{array}{c}
G(j \omega) \\
I
\end{array}\right]^{*} \Pi(j \omega)\left[\begin{array}{c}
G(j \omega) \\
I
\end{array}\right] \leq-\eta I
$$

(ii) for all $\omega \in[0, \infty] \backslash \mathcal{Q}, \tau \in[0,1]$,

$$
\left[\begin{array}{c}
I \\
\tau \Delta(j \omega)
\end{array}\right]^{*} \Pi(j \omega)\left[\begin{array}{c}
I \\
\tau \Delta(j \omega)
\end{array}\right] \geq 0 .
$$

\section{NEGATIVE IMAGINARY TRANSFER FUNCTIONS WITH} IMAGINARY-AXIS POLES

IQC-based conditions for feedback stability of negative imaginary systems with imaginary-axis poles are established in this section. The proofs rely on the arguments detailed in Section II and the generalised IQC result in Section III.

Definition 4.1 ([6, Def. 1]): An $R \in \mathcal{R}^{n \times n}$ is said to be negative imaginary if

(i) $R(s)$ has no poles in $\Re[s]>0$ and at $s=0$;

(ii) $j\left[R(j \omega)-R(j \omega)^{*}\right] \geq 0$ for all $\omega \in(0, \infty)$ except values $\omega$ where $j \omega$ is a pole of $R(s)$;

(iii) if $s=j \omega_{0}$ with $\omega_{0} \in(0, \infty)$ is a pole of $R(s)$, then it is a simple pole and the residue matrix $\lim _{s \rightarrow j \omega_{0}}(s-$ $\left.j \omega_{0}\right) j R(s)$ is Hermitian and positive semidefinite.

Denote by $\mathbf{N}$ the set of negative imaginary transfer functions. Notice that $\mathbf{N}_{s} \subset \hat{\mathbf{N}} \subset \mathbf{N}$.

Theorem 4.2: Given $\bar{G} \in \mathbf{N}_{s}$ and $G \in \mathbf{N}$, suppose that for all $\tau \in[0,1]$,

$$
\begin{gathered}
{\left[\begin{array}{c}
\bar{G}(0) \\
I
\end{array}\right]^{*} \Pi_{0}\left[\begin{array}{c}
\bar{G}(0) \\
I
\end{array}\right]<0 ;} \\
{\left[\begin{array}{c}
I \\
\tau G(0)
\end{array}\right]^{*} \Pi_{0}\left[\begin{array}{c}
I \\
\tau G(0)
\end{array}\right] \geq 0}
\end{gathered}
$$

and

$$
\begin{gathered}
{\left[\begin{array}{c}
\bar{G}(\infty) \\
I
\end{array}\right]^{*} \Pi_{\infty}\left[\begin{array}{c}
\bar{G}(\infty) \\
I
\end{array}\right]<0 ;} \\
{\left[\begin{array}{c}
I \\
\tau G(\infty)
\end{array}\right]^{*} \Pi_{\infty}\left[\begin{array}{c}
I \\
\tau G(\infty)
\end{array}\right] \geq 0,}
\end{gathered}
$$

and for every $j \omega_{0}, \omega_{0} \neq 0$, that is a pole of $G$, the residue matrix $\lim _{s \rightarrow j \omega_{0}}\left(s-j \omega_{0}\right) j G(s)$ is positive definite. Under these conditions, the feedback interconnection $[\bar{G}, G]$ is internally stable.

Proof: The same arguments in the proof for Theorem 2.1 can be used to establish (7) for all $\tau \in[0,1]$ and $\omega \in[0, \infty] \backslash \mathcal{Q}$, where $j \mathcal{Q}$ denotes the set of imaginaryaxis poles of $G$. The only additional requirement is that $\underline{\Omega}$ needs to be sufficiently small and $\bar{\Omega}$ sufficiently large so that $j \mathcal{Q} \cap[0, \underline{\Omega}]=\{\}$ and $j \mathcal{Q} \cap[\bar{\Omega}, \infty]=\{\}$. By Proposition 3.1, this then implies that the closed-loop transfer function

$$
H:=\left[\begin{array}{cc}
I & -G \\
-\bar{G} & I
\end{array}\right]^{-1}
$$

has no poles on $\overline{\mathbb{C}}_{+} \backslash j \mathcal{Q}$. In what follows, we show that $H$ has also no poles in $j \mathcal{Q}$, which then implies $H \in \mathcal{R H}_{\infty}$, 
i.e. the feedback interconnection $[\bar{G}, G]$ is stable.

First note that $\bar{G} \in \mathbf{N}_{s}$ implies $\operatorname{det}(\bar{G}(j \omega)) \neq 0$ for $\omega \neq$ 0 . To see this, observe that if $\operatorname{det}\left(\bar{G}\left(j \omega_{0}\right)\right)=0$ for some $\omega_{0} \neq 0$, then there exists $v \in \mathbb{C}^{n}$ such that $\bar{G}\left(j \omega_{0}\right) v=0$. It follows that $v^{*} j\left[\bar{G}\left(j \omega_{0}\right)-\bar{G}\left(j \omega_{0}\right)^{*}\right] v=0$, which violates the supposition that $\bar{G} \in \mathbf{N}_{s}$. As such,

$$
\operatorname{det}(\bar{G}(j \omega)) \neq 0 \forall \omega \in(0, \infty) .
$$

This implies that there is no closed right-half plane polezero cancellation in the product $G(s) \bar{G}(s)$, since $G \in \mathbf{N}$ has no poles at the origin. Therefore, by [13, Thm. 5.7], $j \omega_{0}$, $\omega_{0} \in \mathbb{R}$ is a pole of $H(s)$ if, and only if, $j \omega_{0}$ is a pole of $(I-G(s) \bar{G}(s))^{-1}$.

Now suppose $j \omega_{0}, \omega_{0}>0$ is an imaginary-axis pole of $G \in \mathbf{N}$, i.e. $j \omega_{0} \in j \mathcal{Q}$. Since $N$ is rational, this is equivalent to $-j \omega_{0} \in j \mathcal{Q}$. In this case, $G(s)$ can be factored as $G(s)=$ $\left((s+\alpha)^{2} /\left(s^{2}+\omega_{0}^{2}\right)\right) G_{1}(s)$, where $\alpha:=\sigma-j \omega_{0}$ and $\sigma>0$ is such that $\alpha$ is not a pole or zero of $N$. Note that $G_{1}$ is proper, rational with complex coefficients. By hypothesis, it follows that

$$
\begin{aligned}
0 & <\lim _{s \rightarrow j \omega_{0}}\left(s-j \omega_{0}\right) j G(s) \\
& =\lim _{s \rightarrow j \omega_{0}}\left(s-j \omega_{0}\right) j \frac{(s+\alpha)^{2}}{s^{2}+\omega_{0}^{2}} G_{1}(s) \\
& =\frac{\alpha^{2}}{2 \omega_{0}} G_{1}\left(j \omega_{0}\right),
\end{aligned}
$$

whereby $G_{1}\left(j \omega_{0}\right)>0$. Observe that

$$
\begin{aligned}
& (I-G(s) \bar{G}(s))^{-1} \\
= & \frac{s^{2}+\omega_{0}^{2}}{(s+\alpha)^{2}}\left(\frac{s^{2}+\omega_{0}^{2}}{(s+\alpha)^{2}} I-G_{1}(s) \bar{G}(s)\right)^{-1} \\
= & : \frac{s^{2}+\omega_{0}^{2}}{(s+\alpha)^{2}} S(s)^{-1} .
\end{aligned}
$$

By combining the fact that $G_{1}\left(j \omega_{0}\right)>0$ and $\operatorname{det}\left(\bar{G}\left(j \omega_{0}\right)\right) \neq$ 0 as shown in (10), we get

$$
\begin{aligned}
\operatorname{det}\left(S\left(j \omega_{0}\right)\right) & =\operatorname{det}\left(G_{1}\left(j \omega_{0}\right) \bar{G}\left(j \omega_{0}\right)\right) \\
& =\operatorname{det}\left(G_{1}\left(j \omega_{0}\right)\right) \operatorname{det}\left(\bar{G}\left(j \omega_{0}\right)\right) \neq 0 .
\end{aligned}
$$

Invoking [13, Lem. 3.38] yields that $S(s)$ has no pole at $j \omega_{0}$. This implies that $(I-G(s) \bar{G}(s))^{-1}$, and hence $H(s)$, has no pole at every $j \omega_{0} \in j \mathcal{Q}$.

All in all, the closed-loop transfer function $H(s)$ in (9) has no poles on $\overline{\mathbb{C}}_{+}$, i.e. $H \in \mathcal{R} \mathbf{H}_{\infty}$ and the feedback interconnection $[\bar{G}, G]$ is stable.

Corollary 4.3: Given $\bar{G} \in \mathbf{N}_{s}$ and $G \in \mathbf{N}$, suppose $\bar{\sigma}(G(0) \bar{G}(0))<1, \bar{\sigma}(G(\infty) \bar{G}(\infty))<1$, and for every $j \omega_{0}$, $\omega_{0} \neq 0$, that is a pole of $G$, the residue matrix $\lim _{s \rightarrow j \omega_{0}}(s-$ $\left.j \omega_{0}\right) j G(s)$ is positive definite. Under these conditions, the feedback interconnection $[\bar{G}, G]$ is internally stable.

Proof: The same arguments in the proof for Corollary 2.3 can be applied to show that the conditions in Theorem 4.2 hold, which implies the claim.

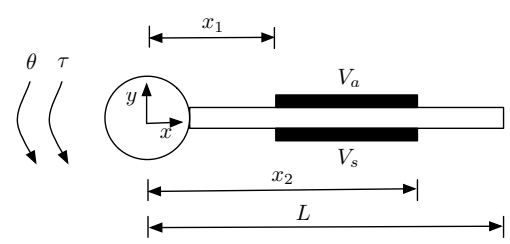

Fig. 2. Schematic diagram of a slewing beam equivalent to a robotic arm.

\section{NUMERICAL EXAMPLES}

Consider a robotic arm pinned to a motor at one end and an equivalent slewing beam model shown in Figure 2; see [14]. Two piezoelectric patches are attached to the arm on either side of the beam. They act as an actuator and a sensor, respectively. The system has input voltage $V_{a}$ applied to the piezoelectric actuator and input torque $\tau$ applied by the motor. On the other hand, the outputs of the system are the voltage $V_{s}$ produced by the piezoelectric sensor and the motor hub angle $\theta$. A distributed-parameter transfer function matrix for the robotic arm is provided in [14]:

$$
\left[\begin{array}{cc}
\frac{N_{\tau, \theta}(s)}{D(s)} & \frac{N_{V_{a}, \theta}(s)}{D(s)} \\
\frac{N_{\tau, V_{s}}(s)}{D(s)} & \frac{N_{V_{a}, V_{s}}(s)}{D(s)}
\end{array}\right]
$$

where $N_{\tau, \theta}, N_{V_{a}, \theta}, N_{\tau, V_{s}}, N_{V_{a}, V_{s}}$, and $D$ are given in equations (26)-(28) in [14].

By approximating the distributed-parameter model as in [7] with a first-resonant mode and ignoring the free body dynamics, one obtains

$$
G(s):=\frac{1}{6.6667 \times 10^{-8}}\left[\begin{array}{cc}
\frac{3.0907}{s^{2}+3.4^{2}}+0.3 & \frac{3.557 \times 10^{-4}}{s^{2}+3.4^{2}} \\
\frac{3.557 \times 10^{-4}}{s^{2}+3.4^{2}} & \frac{2.35}{s^{2}+3.4^{2}}+0.3
\end{array}\right]
$$

Note that $G$ is negative imaginary since $j[G(j \omega)-$ $\left.G(j \omega)^{*}\right]=0$ for all $\omega \in(0, \infty) \backslash 3.4$. This follows from the fact that $G(j \omega)$ is real and symmetric for all $\omega>0$ such that $j \omega$ is not a pole of $G$. Furthermore, the residue matrix

$$
\begin{aligned}
& \lim _{s \rightarrow j 3.4}(s-j 3.4) j G(s) \\
= & \frac{1}{6.6667 \times 10^{-8}}\left[\begin{array}{ll}
\frac{3.0907}{6.8}+0.3 & \frac{3.557 \times 10^{-4}}{6.8} \\
\frac{3.557 \times 10^{-4}}{6.8} & \frac{2.35}{6.8}+0.3
\end{array}\right]>0 .
\end{aligned}
$$

To stabilise the plant $G \in \mathbf{N}$, an integral resonant controller (IRC) [2] is employed. An IRC is a first-order controller taking the form

$$
\bar{G}(s)=(s I+\Gamma \Phi)^{-1} \Gamma-\Delta,
$$

which is strictly negative imaginary if $\Gamma>0, \Phi>0$ and $\Delta$ is symmetric [2, Thm. 8]. Let

$$
\begin{aligned}
\Gamma: & =\left[\begin{array}{ll}
35 & 15 \\
15 & 20
\end{array}\right] \quad \Phi:=\left[\begin{array}{cc}
745 & 521 \\
521 & 1.021
\end{array}\right] \\
\Delta & :=\left[\begin{array}{cc}
2.0871 & -1.0650 \\
-1.0650 & 1.5229
\end{array}\right] .
\end{aligned}
$$

Note that since $\bar{G}(\infty)<0$ and $G(\infty) \bar{G}(\infty) \neq 0$, [6, Thm. 1] cannot be applied here to analyse the stability of the feedback interconnection $[\bar{G}, G]$. However, it can be easily verified 
that $\bar{\sigma}(G(0) \bar{G}(0))=0$ and $\bar{\sigma}(G(\infty) \bar{G}(\infty))=0.8720<1$, whereby Corollary 4.3 holds and $[\bar{G}, G]$ is stable.

Suppose now $\Delta:=\left[\begin{array}{cc}10 & 0 \\ 0 & 10\end{array}\right]$. In this case, $\bar{\sigma}(G(0) \bar{G}(0))=$ $3.3574 \times 10^{7}$ and $\bar{\sigma}(G(\infty) \bar{G}(\infty))=3$. As such, the conditions of Corollary 4.3 fail to hold and hence it cannot be used to conclude stability of $[\bar{G}, G]$. However, by defining

$$
\Pi_{0}=\Pi_{\infty}:=\left[\begin{array}{ll}
0 & I \\
I & 0
\end{array}\right],
$$

it is straightforward to verify that the conditions in Theorem 4.2 hold. As such, $[\bar{G}, G]$ is stable. Note that the multipliers $\Pi_{0}$ and $\Pi_{\infty}$ employed in this example correspond to an IQC characterising passivity [8]. Intuitively, stability of $[\bar{G}, G]$ is established in this example by exploiting the fact that $\bar{G}$ and $G$ exhibit negative imaginary property at positive frequencies that are bounded away from zero and infinity, and positive real property when the frequencies are sufficiently small or sufficiently large.

\section{Conclusions}

This paper establishes sufficient conditions for robust stability of feedback interconnections of negative imaginary systems using an integral quadratic constraint (IQC) approach. Of future interest are generalisations to accommodate free body dynamics corresponding to poles at the origin [7]. Nonlinear systems exhibiting counterclockwise input-output dynamics [15] may also be considered within the framework of IQCs as extensions of negative imaginary systems to nonlinear settings.

\section{REFERENCES}

[1] A. Lanzon and I. R. Petersen, "Stability robustness of a feedback interconnection of systems with negative imaginary frequency response," IEEE Trans. Autom. Contr, vol. 53, no. 4, pp. 1042-1046, 2008.

[2] I. R. Petersen and A. Lanzon, "Feedback control of negative imaginary systems," IEEE Control System Magazine, vol. 30, no. 5, pp. 54-72, 2010.

[3] B. Bhikkaji and S. O. R. Moheimani, "Fast scanning using peizoelectric tube nanopositioners: A negative imaginary approach," in Proc. IEEE/ASME Int. Conf. Advanced Intelligent Mechatronics AIM, Singapore, 2009, pp. 274-279.

[4] B. D. O. Anderson and S. Vongpanitllerd, Network Analysis and Synthesis: A Modern Systems Theory Approach. Prentice Hall, 2007.

[5] S. Devasia, E. Eleftheriou, and S. O. R. Moheimani, "A survey of control issues in nanopositioning," IEEE Transactions on Control Systems Technology, vol. 15, no. 5, pp. 802-823, 2007.

[6] J. Xiong, I. R. Petersen, and A. Lanzon, "A negative imaginary lemma and the stability of interconnections of linear negative imaginary systems," IEEE Trans. Autom. Contr., vol. 55, no. 10, pp. 2342-2347, 2010.

[7] M. A. Mabrok, A. G. Kallapur, I. R. Petersen, and A. Lanzon, "Generalizing negative imaginary systems theory to include free body dynamics: Control of highly resonant structure with free body motion," IEEE Trans. Autom. Contr., vol. 59, no. 10, pp. 2692-2707, 2014.

[8] A. Megretski and A. Rantzer, "System analysis via integral quadratic constraints," IEEE Trans. Autom. Contr., vol. 42, no. 6, pp. 819-830, 1997.

[9] A. Megretski, U. T. Jönsson, C.-Y. Kao, and A. Rantzer, "Integral quadratic constraints," in The Control Handbook, 2nd ed., W. Levine, Ed. CRC Press (Taylor and Francis Group), 2010.

[10] S. Z. Khong, E. Lovisari, and A. Rantzer, "A unifying framework for robust synchronisation of heterogeneous networks via integral quadratic constraints," IEEE Trans. Autom. Contr., 2014, submitted. [Online]. Available: http://www.control.lth.se/media/Staff/ EnricoLovisari/SynchUnifiedIQCKhongLovisariRantzerPreprint.pdf
[11] S. K. Das, H. R. Pota, and I. R. Petersen, "Stability analysis for interconnected systems with mixed passivity, negative-imaginary and small-gain properties," in Australian Control Conference, Perth, Australia, 2013.

[12] M. Cantoni, U. T. Jönsson, and C.-Y. Kao, "Robustness analysis for feedback interconnections of unstable distributed systems via integral quadratic constraints," IEEE Trans. Autom. Contr., vol. 57, pp. 302$317,2012$.

[13] K. Zhou, J. C. Doyle, and K. Glover, Robust and Optimal Control. Upper Saddle River, NJ: Prentice-Hall, 1996.

[14] H. R. Pota and T. E. Alberts, "Multivariable transfer functions for a slowing piezoelectric laminate beam," Journal of Dynamic Systems, Measurements and Control, vol. 117, no. 2, pp. 352-359, 1995.

[15] D. Angeli, "Systems with counterclockwise input-output dynamics," IEEE Trans. Autom. Contr., vol. 51, no. 7, pp. 1130-1143, 2006. 